$16^{\text {th }}$ International Congress of Metrology, 01001 (2013)

DOI: $10.1051 /$ metrology/201301001

(c) Owned by the authors, published by EDP Sciences, 2013

\title{
The new METAS Watt Balance, Mark II
}

\author{
H. Baumann ${ }^{1}$, A. Eichenberger ${ }^{1}$, F. Cosandier ${ }^{1}$, B. Jeckelmann ${ }^{1}$, R. Clavel $^{3}$, D. Reber ${ }^{2}$, D. Tommasini ${ }^{4}$, C. Hajdu $^{4}$ \\ ${ }^{1}$ Federal Institute of Metrology METAS, CH-3003 Bern-Wabern, Switzerland. \\ ${ }^{2}$ METTLER TOLEDO, CH-8606 Nänikon, Switzerland \\ ${ }^{3}$ Laboratoire de Systèmes Robotiques, LSRO, Ecole Polytechnique de Lausanne, EPFL, CH-1015 Lausanne, Switzerland \\ ${ }^{4}$ Centre Européen de Recherche Nucléaire, CERN, CH-1211 Genève, Switzerland
}

\begin{abstract}
In the context of the future new definition of the mass unit, $\mathrm{kg}$, a promising route to link the mass unit to a fundamental constant, the Planck constant $h$, is represented by the watt balance experiment. Since a number of years, the Federal Institute of Metrology is active in this field and published a first result with its Mark I experiment in 2011. To reach the target uncertainty of 2 parts in $10^{8}$, the Mark II METAS watt balance is being developed. In this paper, the different modules of the experiment are presented and their respective metrological requirements and experimental behaviour are discussed.
\end{abstract}

\section{Introduction}

The definition of the unit of mass, the kilogram, still corresponds to the one formulated more than hundred years ago. Today, the $\mathrm{kg}$ is the last base unit in the International System of Units (SI) based on an artefact, the International Prototype of the Kilogram (IPK). In view of the results of the three international comparisons between IPK and the national copies, it is nowadays widely accepted that the mass unit should be redefined. Linking the mass unit to the Planck constant $h$ by means of the watt balance experiment is one possible experimental approach pursued by a number of institutes [1].

After several years of development, characterisation and continuous improvement of its first watt balance, METAS published a final value of the Planck constant [2]. Based on the acquired experience, the main modules of the METAS watt balance have been newly designed in collaboration with specialized partners [3]. The translation stage has been developed in close collaboration with the Ecole Polytechnique de Lausanne EPFL, the magnetic circuit has been realized with the group of Magnets and Superconductors from CERN and finally, a completely new load cell based on the monolithic technology has been designed by MettlerToledo. An overview of the new design is given in figure 1 .

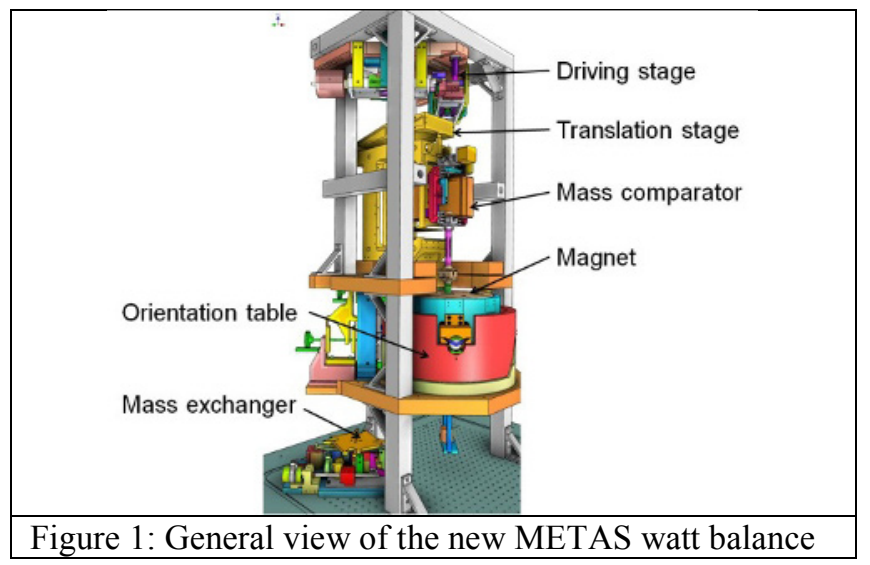

\section{Working principle}

The concept of the watt balance is discussed in detail elsewhere [1]. The experiment is performed in two steps with the same experimental setup: the static or weighing mode and the dynamic or induction mode (figure 2).

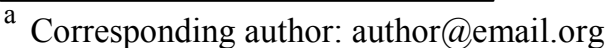




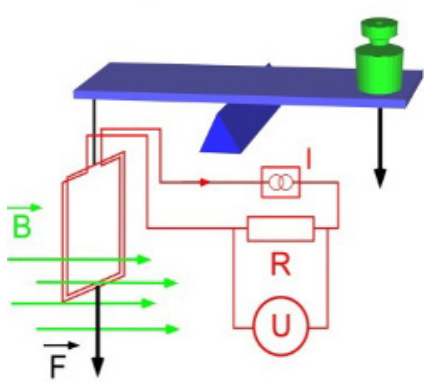

Static mode

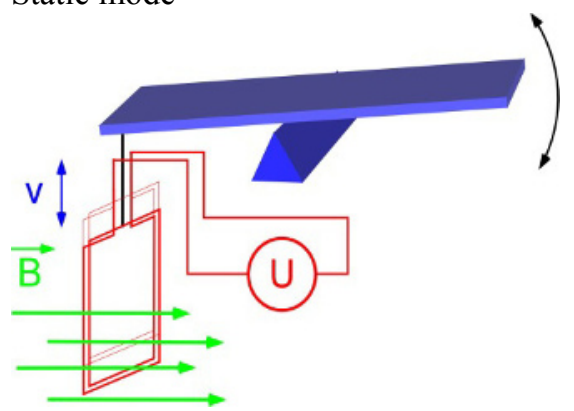

Dynamic mode.

Figure 2: Principle of the watt balance experiment.

In the static mode, the force generated by a mass $m$ placed in the local gravity field $g$, is balanced by the vertical component of the electromagnetic force produced by a current $I$ flowing in a coil immersed in a magnetic field of flux density $\boldsymbol{B}$. The electromagnetic force can be expressed by

$\vec{F}=I \cdot \oint d \vec{l} \times \vec{B}$

where $\boldsymbol{d} \boldsymbol{l}$ is the elementary conductor length of the coil. In the dynamic mode, the coil is moved vertically at a velocity $v$ though the magnetic flux $\boldsymbol{B}$. This motion induces a voltage $U$ across the coil that can be expressed by

$U=\oint(\vec{v} \times \vec{B}) \cdot d \vec{l}=-\oint(d \vec{l} \times \vec{B}) \cdot \vec{v}$

If the magnetic field and the mechanical dimensions of the coil are strictly identical in both modes, and under the hypothesis that the coil passes through its weighing position during the velocity mode with the same orientation, the combination of both modes leads to the expression

$U \cdot I=m \cdot g \cdot v$

The experiment thus allows a virtual comparison of the electrical and the mechanical power. Using the expressions for the Josephson and quantum Hall effects, equation (3) can be rewritten as

$m=C \cdot \frac{f_{J} \cdot f_{J}^{\prime}}{g \cdot v} \cdot h$, where $C$ is a dimensionless calibration factor, $f_{J}$ and $f_{J}$, are the Josephson frequencies used during the static and the dynamic phase and $h$ the Planck constant. The watt balance experiment thus relates the unit of mass to the meter, the second and the Planck constant.

\section{The translation and driving stage}

The translation of the coil along a vertical path is a crucial operation in a watt balance experiment. In close collaboration with the laboratory of robotics systems of the EPFL a 13-hinge translation stage has been developed.

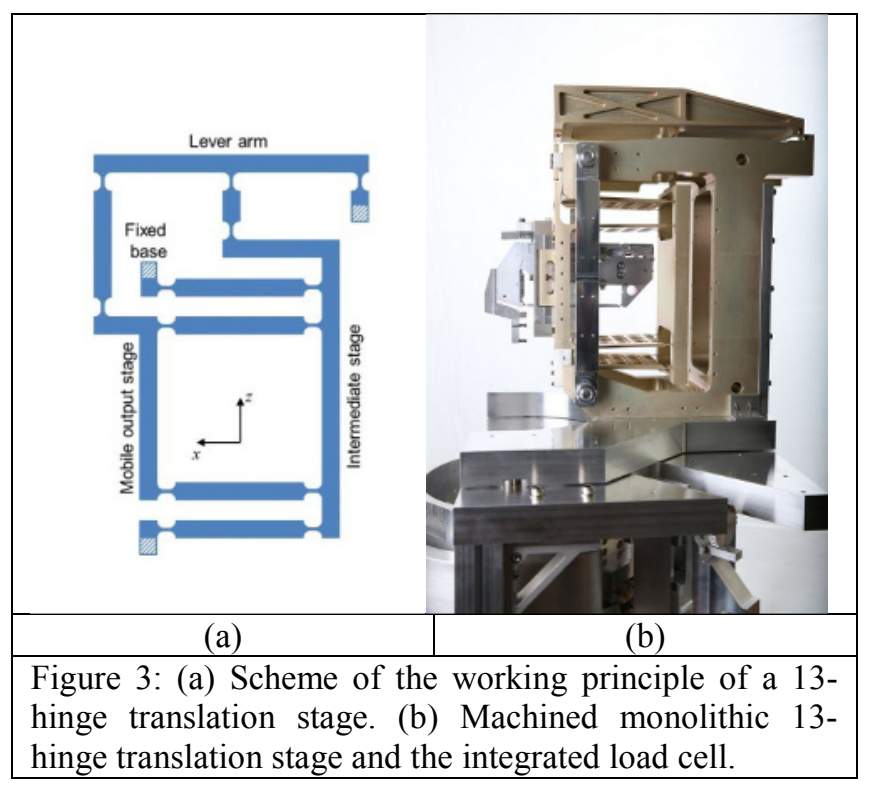

Figure 3 gives a schematic overview of the working principle of the 13-hinge stage. A coupling lever links the output stage to an intermediate stage. By an optimized design, the non-linear motion of the output stage can by reduced by the asymmetric motion of the intermediate stage [4]. The straightness of the outlet has been measured with an interferometer. Over a total displacement of $40 \mathrm{~mm}$, the peak to peak deviations from the straightness have been evaluated to $200 \mathrm{~nm}$ in $x$ and $40 \mathrm{~nm}$ in $y$.

During the dynamic phase of the experiment, the translation stage, the comparator and the complete suspension is moved at a velocity of a few $\mathrm{mm} / \mathrm{s}$. The working principle of the driving stage is based on a Sarrus linkage [4]. It has been designed to compensate the stiffness of the translation stage as well as the weight of the whole suspension. By this way, the deviation of the verticality in $x$ and $y$ directions could be reduced to better than $2 \mu \mathrm{m}$ and the maximal force needed by the motor is approximately $30 \mathrm{mN}$.

\section{Load cell}

During the static phase of the watt balance experiment, the rest force is measured with a mass comparator 
integrated in the translation stage. The load cell has to satisfy a number of unusual requirements like low mass, robustness, low sensitivity to external disturbance and vacuum compatibility. The prototype developed especially for this project by Mettler Toledo (see figure 2) shows very impressive characteristics like a high repeatability $(\sigma=0.29 \mu \mathrm{g})$ and a fast time response.

\section{Magnetic circuit}

The magnetic circuit, designed in close collaboration with the group of Magnets and Superconductors from the CERN is a closed cylindrical geometry. The magnetic field in the gap is $0.6 \mathrm{~T}$ with a vertical homogeneity of $10^{-5}$. To reduce the temperature dependence of the circuit, the magnetic field is produced by two SmCoGd permanent magnets. The typical temperature coefficient of this alloy is around $-10 \mathrm{ppm} / \mathrm{K}$. The remaining temperature dependence is finally compensated by introducing a well dimensioned magnetic shunt. First experimental measurements have shown that the temperature coefficient of the circuit can be reversed. This implies that the coefficient can be cancelled in theory [3].

\section{Position of the coil}

The determination of the coil position at the point of comparison between the two modes is one of the key parameters of the experiment. To be able to measure all 6 degrees of freedom, a number of optical elements have been fixed to the coil support. A view of the coil, its support structure and the optical elements is given in figure 4 . In addition to the optical elements, three ceramic balls have been placed on the upper part of the coil structure. They can be used as reference points for a tripod serving as external reference plane.

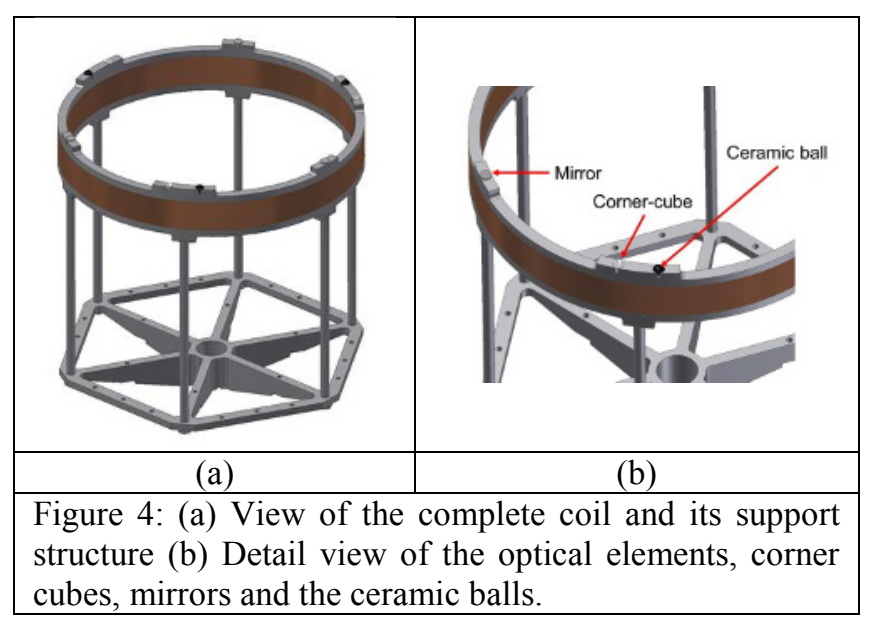

The measurement principle is schematically given in figure 5. The position along the $z$ direction is measured with the interferometer used to measure the velocity in the dynamic mode.

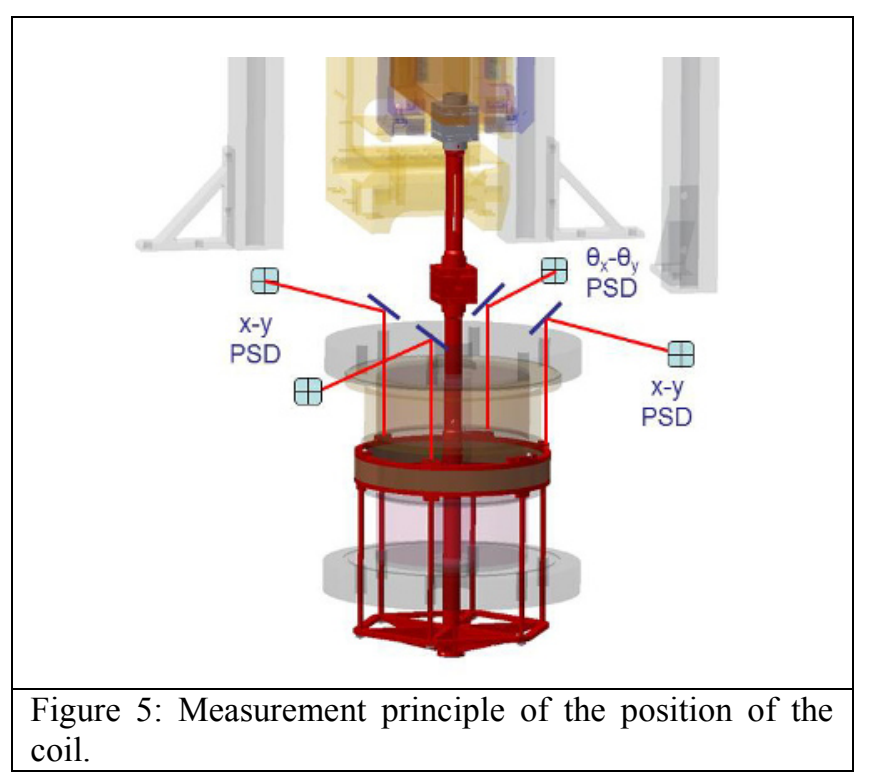

The displacement in the $x-y$ plane is measured with three corner-cube-PSD (Position Sensitive Detector) assemblies. The working principle for this kind of detector is represented in figure 6 . The beam of a laser diode sent through a beam splitter and a quarter wave plate is reflected by a corner cube. The reflected beam is then deflected by the beam splitter to a PSD. The variation of the position measured on the PSD is proportional to the translation of the corner cube. The information obtained by these detectors can then also be used to determine the rotation of the coil around the $z$ axes

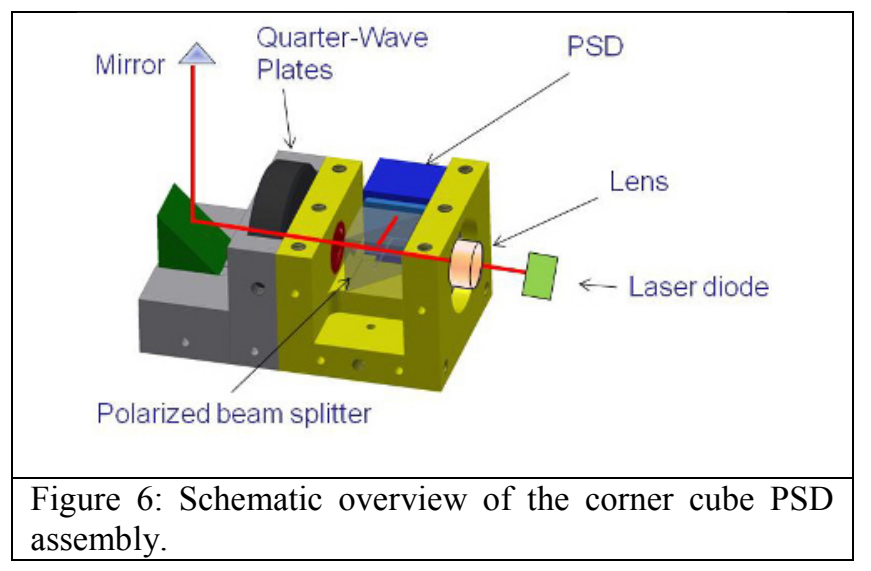

To determine the angles around the $x$ and $y$ axes, an autocollimator has been developed (figure 7) [5]. Preliminary measurements have shown an uncertainty of a few $\mu \mathrm{rad}$ for a range of $1 \mathrm{mrad}$. 


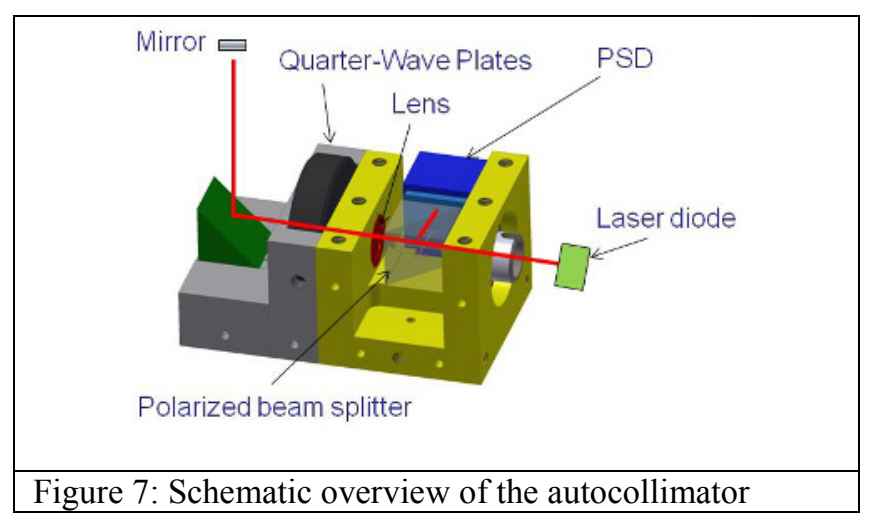

\section{Simulations}

At present, the knowledge of the whole experiment is essentially based on an experimental approach. To improve the understanding of the behaviour of such a complex experiment, several simulations will be performed and compared to the experimental measurements. A first result has been achieved by comparing the simulated temperature dependence of a test magnetic circuit and the experimental results. This initial simulation step is a first benchmark for the construction of a theoretical model of the experiment that will be a powerful tool to identify critical points of the experiment.

\section{Conclusions}

After the publication of a first value of the Planck constant $h$, the critical modules of the experiment have been newly designed in collaboration with specialized industrial partners, research institutes and universities. The different modules have been individually evaluated and assembled. The characterisation of the complete new METAS watt balance MARK II will be performed during 2013 and the Planck constant determination should start in 2014 .

\section{Acknowledgments}

This work was jointly funded by the European Metrology Research Program (EMRP) participating countries within the European Association of National Metrology Institutes (EURAMET) and the European Union.

\section{References}

1. A. Eichenberger, G. Genevès, and P. Gournay "Determination of the Planck constant by means of a watt balance," Eur. Phys. J. B, vol. 172, pp. 363-383, 2009.

2. A. Eichenberger, H. Baumann, B. Jeanneret, B. Jeckelmann, P. Richard, and W. Beer, "Determination of the Planck constant with the METAS watt balance," Metrologia, vol. 48, pp. 133-141, 2011.
3. H. Baumann, A. Eichenberger, F. Cosandier, B. Jeckelmann, R. Clavel, D. Reber and D. Tommasini, "Design of the new METAS watt balance experiment Mark II" Metrologia, vol. 50, pp. 235-242, 2013.

4. F. Cosandier, Phd Thesis, 'Conception d'Axes Motorisés Rectilignes d'Ultra-Haute Précision', EPFL 2013.

5. W. Gao "Precision nanotechnology: Sensors and Measuring Systems for Nanomanufacturing". ISBN 978-1-84996-254-4. 